\title{
The Scattering Transformation for Networked Control Systems
}

\author{
Tilemachos Matiakis, Sandra Hirche, and Martin Buss
}

\begin{abstract}
Communication time delay in a networked control system (NCS) degrades the performance and may lead to instability. For the first time the scattering transformation is applied to NCS in order to guarantee stability in the presence of arbitrarily large constant time delay. This approach, initially derived from network theory and often used in bilateral teleoperation with time delay, requires in its original version all subsystems to be passive. In this paper a stability condition is derived relaxing this restriction such that also non-passive plants can be stabilized. The performance of the proposed approach is superior over two standard approaches, the Smith predictor and PI control in the presence of time delay uncertainties. Experimental validation results are presented.
\end{abstract}

\section{INTRODUCTION}

With the ongoing development of advanced communication technologies communication networks become more and more attractive for the signal transmission in control systems. In such a networked control system (NCS) the plant and the controller are spatially separated and the control loop is closed physically through the communication network as shown in Fig. 1. Compared to traditional control systems with point to point connections the use of NCS results in a decreased complexity and costs, easier maintenance and system diagnosis, and higher reliability. Until now specific industrial networks, such as CAN and PROFIBUS, have been mainly used in NCS. Recently, common purpose networks, such as e.g. Ethernet-like networks, attract the attention due to their high flexibility.

However, the transmission of the controller and plant output over a communication network introduces a time delay into the closed loop system, which can be constant or varying. In Ethernet-like networks the time delay depends on the concurrent amount of traffic and there are no guarantees on the maximum time delay. It is well-known that time delay in a closed control loop degrades the performance and can lead to instability.

The various stability conditions and the derived stabilizing control methods known in the literature can be separated into two categories, delay-dependent and delay-independent approaches. In the first case an upper bound for the time delay has to be assumed, and stability is guaranteed as long as the time delay remains within this bound, while in the second case stability is ensured for arbitrarily large time delays.

Most of the methods developed for the analysis and design of NCS are delay-dependent. In [1] the time delay

All authors' affiliation: Institute of Automatic Control Engineering, Technische Universität München, D-80290 München, Germany http: //www.lsr.ei.tum.de,

T.Matiakis@tum.de, S.Hirche@ieee.org, M.Buss@ieee.org

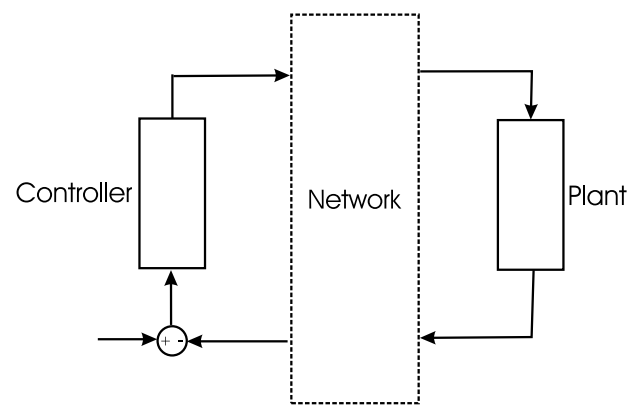

Fig. 1. Networked control system architecture.

is assumed to be bounded by the sampling period and the stability is analyzed using hybrid system techniques. Random time delays, also bounded by the sampling period, are considered in [2], a stochastic stability analysis is performed and stochastic optimal control applied. A delay-dependent approach based on Lyapunov stability with LMI conditions is proposed in [3]. In the seminal work [4], [5] an augmentation technique is applied to transform the time delay system to an augmented discrete system without time delay and a stability condition is presented for periodic time delays. The notion of Maximum Allowable Transfer Interval (MATI) is introduced in [6] denoting the maximum time interval between two successive messages of each sensor for the system to remain stable. A survey on recent NCS control methodologies is presented in [7], further analysis and synthesis methods for time delay systems can be found in [8].

In this paper a delay-independent approach is considered. To the best knowledge of the authors for the first time the scattering transformation is applied to NCS. Aiming at stability of a time delay system this method has been firstly applied to telepresence systems in [9] and extended in [10]. Using the concepts of passivity and network theory, the derived control law is based upon the fact that a system of interconnected subsystems is passive if each of them is passive, and consequently stable. The scattering transformation passivates the communication subsystem with arbitrarily large constant time delay. However, in its original version both, the plant and the controller, are required to be passive. In this paper we will show that under certain conditions a non-passive LTI plant can be stabilized independently of the constant time delay. Furthermore we show that the control performance gracefully degrades with increasing time delay, i.e. is robust with respect to time delay variations and uncertainties.

This paper is organized as follows: Section II introduces the notions of passivity, positive real systems and scattering 
transformation; a novel stability condition for non-passive plants is derived in Section III followed by design aspects and a performance comparison with standard approaches in Section IV and experiments in Section V.

\section{BACKGROUND}

\section{A. Passive and Positive Real Systems}

The passivity property of a system's element is a useful tool for its stability analysis. An element is said to passive if it does not generate energy, which means that given zero energy storage at $t=0$, the property

$$
\int_{0}^{t} P_{i n}(\tau) \mathrm{d} \tau=\int_{0}^{t} u^{T}(\tau) y(\tau) \mathrm{d} \tau \geq 0 \quad \forall t>0,
$$

holds, with $P_{i n}(\tau)$ denoting the instantaneous power input to the system, $u(\tau), y(\tau)$ the input and output vectors. If two passive systems are combined in parallel or feedback connection the resulting system is still passive [11]. Thus, by induction any combination of passive systems in parallel or feedback connection is again passive.

An important practical feature of the passivity formulation is that it can be easily defined in the Laplace domain for linear systems. A stable SISO, LTI system $H(j \omega)$ is passive [11] if and only if

$$
\operatorname{Re}\{H(j \omega)\} \geq 0, \quad \forall \omega \geq 0,
$$

which means that the entire Nyquist plot of the system lies in the right half plane. Another notion related with passivity is positive realness. A SISO system $H(s)$ is said to be positive real $(\mathrm{PR})$ if

$$
\operatorname{Re}\{H(s)\} \geq 0, \quad \forall \sigma \geq 0,
$$

where $s=\sigma+j \omega$ is the Laplace variable. A system is passive if and only if it is PR. Thus, the notions of passivity and positive realness can be interchanged indiscriminately. Further, a system $H(s)$ is strictly positive real (SPR) if $H(s-\varepsilon)$ is PR for some $\varepsilon>0$. Necessary conditions for a SPR system is that it is strictly stable and

$$
\operatorname{Re}\{H(j \omega)\}>0, \quad \forall \omega \geq 0,
$$

i.e $H(s)$ is strictly positive on the $j \omega$-axis. The major difference between PR and SPR is that the first can tolerate poles on the imaginary axis as long as they are simple and the associated residues are real and non-negative.

\section{B. Scattering Transformation}

In case of time delay in the communication network the bidirectional communication channel can be modeled as a time delaying two-port with time delays $T_{1}, T_{2}$ in the forward and the backward path respectively, as shown in Fig. 2. The block $H_{p}$ denotes the plant and $H_{c}$ the controller, both assumed to be SISO LTI systems. The blocks of the scattering transformation are explained below.

The time-delaying two-port generates energy as shown in [9], i.e. the passivity condition is violated. The scattering

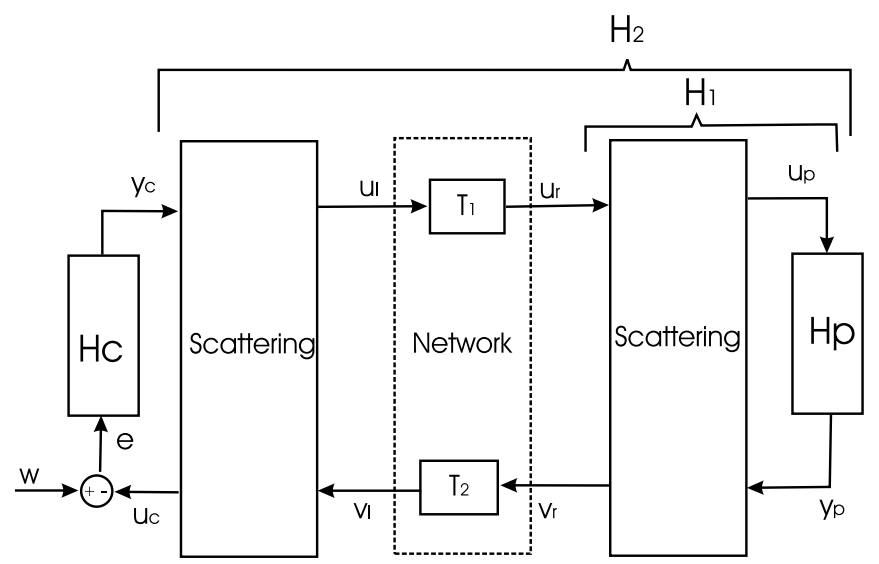

Fig. 2. NCS with scattering transformation.

transformation is used in order to passivate the communication network with the transformation equations given by

$$
\begin{aligned}
& u_{l}=\frac{1}{\sqrt{2 b}}\left(u_{c}+b y_{c}\right) ; \quad u_{r}=\frac{1}{\sqrt{2 b}}\left(y_{p}+b u_{p}\right) ; \\
& v_{l}=\frac{1}{\sqrt{2 b}}\left(u_{c}-b y_{c}\right) ; \quad v_{r}=\frac{1}{\sqrt{2 b}}\left(y_{p}-b u_{p}\right)
\end{aligned}
$$

where $b>0$ is a tuning parameter that can be chosen freely. Furthermore, the relation between the left and right hand scattering variables is as follows $u_{r}(t)=u_{l}\left(t-T_{1}\right)$ and $v_{l}(t)=v_{r}\left(t-T_{2}\right)$.

The energy balance (1) for the two-port is now computed with the input vector $u^{T}=\left[y_{c}-y_{p}\right]$ and the output vector $y^{T}=\left[\begin{array}{ll}u_{c} & u_{p}\end{array}\right]$, see Fig. 2 and the reformulated transformation equations (4) inserted

$$
\begin{aligned}
\int_{0}^{t} P_{i n}(\tau) \mathrm{d} \tau & =\int_{0}^{t}\left(u_{c} y_{c}-u_{p} y_{p}\right) \mathrm{d} \tau \\
& =\frac{1}{2} \int_{0}^{t}\left(u_{l}^{2}-u_{r}^{2}+v_{r}^{2}-v_{l}^{2}\right) \mathrm{d} \tau \\
& =\frac{1}{2} \int_{t-T_{1}}^{t} u_{l}^{2} \mathrm{~d} \tau+\frac{1}{2} \int_{t-T_{2}}^{t} v_{r}^{2} \mathrm{~d} \tau \geq 0 \quad \forall t .
\end{aligned}
$$

The passivity condition (1) is satisfied, hence the communication two-port with constant time delay is passive, in fact it is energetically lossless as the energy is stored in the communication two-port for the time of transit and released afterwards.

\section{StABILITY}

\section{A. Stability with a Passive Plant and Controller}

If the plant is passive, i.e. satisfies (1), then the subsystem $\mathrm{H}_{2}$ comprising the plant and the communication subsystem including the scattering transformation, see Fig. 2 is passive. Further, the controller $H_{c}$ is assumed to be passive. The two passive subsystems $H_{2}$ and $H_{c}$ are in feedback interconnection, thus the closed control loop is passive and consequently, under observability assumptions, stable. In 
fact, all the passivity theorems [11] and the corresponding stability results can be applied. The controller and the plant can be either linear or non-linear, continuous or discrete time, time-invariant or time-varying subsystems. The plant parameters can be even uncertain as long as the plant remains passive. However, the passivity requirements might be too restrictive in real applications. In the following, a sufficient condition for the stability is derived relaxing these requirements.

\section{B. Stability with a Non-Passive Plant or Controller}

In this first approach the continuous time case is considered, the time delays $T_{1}, T_{2}$ are assumed to be constant but arbitrarily large, the plant and the controller are LTI systems, the scattering transformation (4) is applied with the structure of the closed loop system presented in Fig. 2.

Theorem: If

$$
K(s)=\frac{1}{b} \frac{b^{2} H_{c}+H_{p}}{1+H_{c} H_{p}},
$$

where $H_{p}$ denotes the plant transfer function, $H_{c}$ the controller transfer function, and $b$ the tuning parameter of the scattering transformation, is PR, i.e.

$$
\operatorname{Re}\{K(s)\} \geq 0, \quad \forall \sigma \geq 0,
$$

then the closed loop system is stable independently of the time delay. If condition (7) holds strictly, that is $K(s)$ is SPR (3), then the closed loop system is asymptotically stable.

Proof: The proof is straightforward and follows from computing the roots $s_{r}$ of the closed loop system. It is shown that as long as $K(s)$ is PR then the system has no roots in the open right half plane.

From the reformulated equations of the scattering transformation (4), the open loop transfer function $H_{O L}$ of the system, i.e. the transfer function from the controller input $e$ to the output of the left part of the scattering transformation $u_{c}$ in Fig. 2 is computed

$$
H_{O L}(s)=b H_{c}(s) \frac{1+e^{-s T} H_{1}(s)}{1-e^{-s T} H_{1}(s)},
$$

with $T=T_{1}+T_{2}$ the roundtrip time delay in the communication network and

$$
H_{1}(s)=\frac{H_{p}(s)-b}{H_{p}(s)+b},
$$

the transfer function from $u_{r}$ to $v_{r}$ computed by means of (4).

The roots $s_{r}$ of the closed loop system are placed where the equation $H_{O L}\left(s_{r}\right)=-1$ holds, thus substituting $H_{1}$ (9) in (8) yields

$$
H_{O L}\left(s_{r}\right)=b H_{c}\left(s_{r}\right) \frac{b+H_{p}\left(s_{r}\right) \frac{1+e^{-s_{r} T}}{1-e^{-s_{r} T}}}{b \frac{1+e^{-s_{r} T}}{1-e^{-s_{r} T}}+H_{p}\left(s_{r}\right)}=-1,
$$

and after some mathematical manipulation

$$
-\frac{1+e^{-s_{r} T}}{1-e^{-s_{r} T}}=\frac{1}{b} \frac{b^{2} H_{c}\left(s_{r}\right)+H_{p}\left(s_{r}\right)}{1+H_{c}\left(s_{r}\right) H_{p}\left(s_{r}\right)}=K\left(s_{r}\right) .
$$

Now considering the real part of the transfer function $K\left(s_{r}\right)$ that is defined according to (6), at the roots $s_{r}$ of the closed system the equation

$$
\begin{aligned}
& \operatorname{Re}\left\{K\left(s_{r}\right)\right\}=\operatorname{Re}\left\{-\frac{1+e^{-s_{r} T}}{1-e^{-s_{r} T}}\right\} \\
= & -\operatorname{Re}\left\{\frac{1-e^{-2 \sigma_{r} T}-j 2 e^{-\sigma_{r} T} \sin \omega_{r} T}{\left(1-e^{-\sigma_{r} T} \cos \omega_{r} T\right)^{2}+\left(e^{-\sigma_{r} T} \sin \omega_{r} T\right)^{2}}\right\} \\
= & -\frac{1-e^{-2 \sigma_{r} T}}{\left(1-e^{-\sigma_{r} T} \cos \omega_{r} T\right)^{2}+\left(e^{-\sigma_{r} T} \sin \omega_{r} T\right)^{2}},
\end{aligned}
$$

must hold. Lets assume $K(s)$ to be PR (7). It can be easily seen that in this case there exists no solution $s_{r}$ of (10) for $\sigma_{r}>0$ as the real part of $K\left(s_{r}\right)$ is always non-negative then, while the right part of (10) is negative. Consequently, the closed loop system has no roots in the open right half plane, thus is stable. Furthermore, if $K(s)$ is assumed to be SPR, then (10) has no solution $s_{r}$ on the imaginary axis as well, thus the closed loop system has no roots in the closed right half plane, i.e. is asymptotically stable.

For the control design the tuning parameter $b$ can be chosen such that the stability condition (7) is satisfied.

\section{Simulations}

In order to test the validity of the above condition simulations are conducted. A non-passive plant is considered with the transfer function

$$
H_{p}(s)=\frac{20}{(s+2)(s+10)},
$$

and a PI controller

$$
H_{c}(s)=\frac{1.5(s+2)}{s},
$$

which guarantees a zero steady state error.

The influence of the tuning parameter $b$ on the positive realness of $K(s)(6)$ and thus the system's stability is investigated. From the Nyquist plot of $K(s)$ in Fig. 3 it can be seen that the plot moves towards the right half plane with increasing $b$. For $b=0.2$ it crosses the imaginary axis into the right half plane and continues to move deeper into it. Thus, for $b \geq 0.2, K(s)$ is SPR, and while $b$ increases it becomes more robustly SPR. The step response of the system for different values of time delay $T \in[150,300,1000] \mathrm{ms}$ and different values of $b$ are depicted in Fig. 4. As expected the closed loop system is stable for $b=0.2$. In the first case $(T=150 \mathrm{~ms})$ the system becomes unstable for approximately $b=0.18$ while in the other two cases $(T=300 \mathrm{~ms}$ and $T=1000 \mathrm{~ms})$ at approximately $b=0.16$. In all step responses oscillations can be observed, i.e. for $b \approx 0.2$ there are roots close to the imaginary axis. This indicates that the sufficient stability condition (7) in the considered case is not conservative.

\section{PERFORMANCE}

In the following, controller design aspects are discussed. Main objectives are the achievement of a specified steady state behavior and the robust closed loop performance in the presence of time delay uncertainties. Further, a comparison with standard approaches is performed. 


\section{A. Design Aspects}

The transfer function of the closed loop system is computed from Fig. 2 and the transformation equations (4) to be

$$
H(s)=\frac{Y_{p}(s)}{W(s)}=\frac{H_{c} H_{p}}{1+H_{c} H_{p}} \frac{2 e^{-s T_{1}}}{K\left(1-e^{-s T}\right)+\left(1+e^{-s T}\right)},
$$

where $K=K(s)$ is defined according to (6), $W(s)$ is the desired value, and $Y_{p}(s)$ the output of the plant, see Fig. 2. The closed loop transfer function comprises two parts: the left part is the closed loop transfer function without time delay and scattering transformation, the right part incorporates the effect of the time delay and of the scattering transformation on the closed loop behavior.

Looking at the steady state response $s=0$ the right part in (13) is equal to one

$$
H(0)=\frac{H_{c}(0) H_{p}(0)}{1+H_{c}(0) H_{p}(0)} .
$$

As long as the system satisfies the stability condition (7) the time delay and the scattering transformation do not affect the steady state behavior. Thus, concerning the steady state error, the controller can be designed without considering the time delay and the scattering transformation.

Primary objective is to design a controller which is robust to different values of the time delay. The sensitivity function with respect to the time delay $T$ is

$$
S_{T}^{H^{*}}=\frac{T}{H^{*}} \frac{d H^{*}}{d T}=-\frac{s T e^{-s T}(K-1)}{K\left(1-e^{-s T}\right)+\left(1+e^{-s T}\right)},
$$

where $H^{*}=H e^{s T_{1}}$ represents the closed loop transfer function (13) without the purely time delay shifting part $e^{-s T_{1}}$. As long as $K(s)$ is close to one the sensitivity function is almost zero and consequently the system is robust to time delay uncertainties. Thus, the design objective is to find a
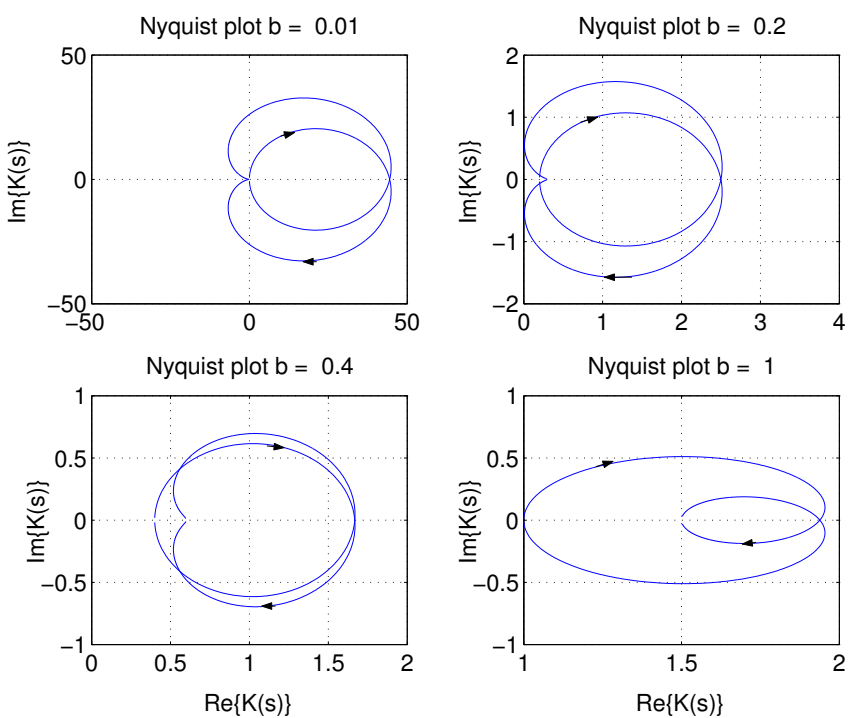

Fig. 3. Nyquist plot of $K(s)$ for different values of $b$, the stability condition (7) is violated for $b=0.01$ (upper left plot), and marginally satisfied for $b=0.2$ (upper right plot).
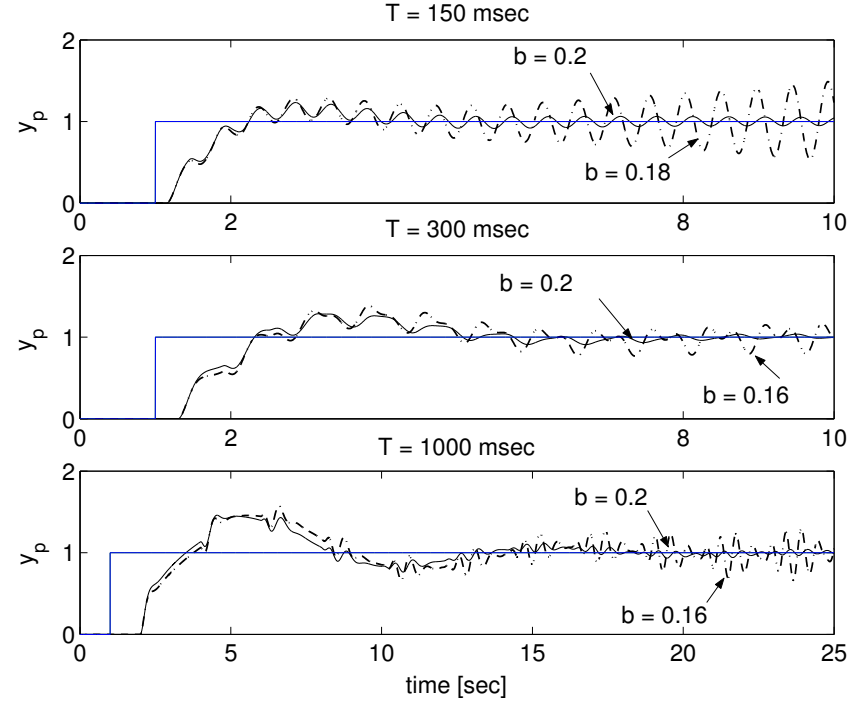

Fig. 4. Step responses for different values of $b$ and roundtrip time delay $T$, stability for $b=0.2$ independent of time delay.

controller and a parameter $b$ such that $K(s) \approx 1$ holds over a broad frequency range. If exactly $K(s)=1$ holds $\forall s$ then (13) reduces to

$$
H(s)=\frac{H_{c}(s) H_{p}(s)}{1+H_{c}(s) H_{p}(s)} e^{-s T_{1}},
$$

which is the closed transfer function with zero time delay in the loop, but the response shifted by the forward time delay $T_{1}$. Concluding the above, the overall design goal is to find a controller and a value for $b$ such that the closed loop system without the time delay and the scattering transformation has a satisfying response while $K(s) \approx 1$ for a broad range of frequencies.

\section{B. Performance Comparison}

The proposed control approach is compared with two common design approaches for systems with time delay, the Smith predictor and PI control. Of specific interest is the performance robustness with respect to time delay uncertainties. Both the compared approaches are delay-dependent, i.e. the time delay has to be known for the control design which is an unreasonable assumption in Ethernet-like networks.

Specifically, the integrating part of the PI controller, ensuring a zero steady state error of the closed loop, inhibits the delay-independent design, as the open loop transfer function then cannot satisfy the delay-independent stability condition $\left|H_{O L}\right|_{\infty}<1$.

In the following a maximum roundtrip time delay of $T=300 \mathrm{~ms}$ is assumed, further, the plant is given by (11). For the scattering approach the controller $H_{c}$ (12) is used, ensuring a zero steady state error as long as the stability condition (7) is satisfied, i.e. $b \geq 0.2$, see Sec. III-C. The parameter $b=1$ is chosen according to the previous considerations such that $K(0)=1$ holds.

For the compared standard approach the PI controller (12) is used with a gain $k>0$ adapted to ensure stability with the 

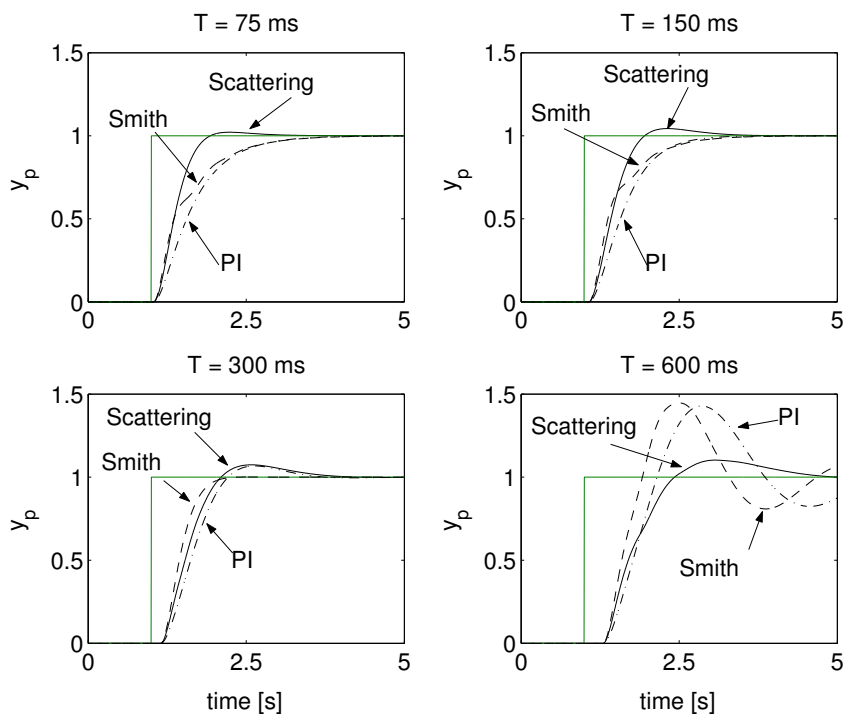

Fig. 5. Step responses of the system with scattering approach, Smith predictor, and PI controller for different value of time delay.

assumed maximum time delay

$$
H_{c}^{P I}(s)=k H_{c}(s)
$$

The gain is tuned heuristically such that a compromise between the rise time and the overshoot is obtained. For $k=0.45$ the overshoot is less than $10 \%$ for $T=300 \mathrm{~ms}$ which is considered to be acceptable in this case.

For the Smith predictor approach the controller $H_{c}$ from (12) is used to design the final controller

$$
H_{c}^{S P}(s)=\frac{H_{c}(s)}{1+H_{c}(s) H_{p}(s)\left[1-e^{-s T}\right]},
$$

where $T=300 \mathrm{~ms}$ represents the maximum time delay. Note, that full knowledge of the plant is required for its design. In case of exact plant and time delay knowledge the response of the time delayed system with Smith predictor equals the response of the system without time delay shifted in the time axis by $T$.

The step responses for all three approaches are compared for different values of time delay, the initially presumed time delay $T=300 \mathrm{~ms}$, and $T \in[75,150,600] \mathrm{ms}$. The results are shown in Fig. 5. As expected for the nominal time delay $T=300 \mathrm{~ms}$ the Smith predictor achieves the best performance with respect to rise time and overshoot. However, with decreasing time delay the rise time of the PI controlled and the Smith predictor system increases substantially, i.e. the performance is degraded, while the controller with the scattering transformation shows an increasingly better performance. If the time delay goes to zero the step response tends towards the response of the system without time delay and no scattering transformation, see (13) for $T=T_{1}=0$. For higher time delays than expected, see Fig. 5 for $T=600 \mathrm{~ms}$, both the Smith predictor and the PI controller give high overshoot and a high settling time, while the performance of the scattering controller deteriorates gracefully.
In summary, the proposed control approach with the scattering transformation is robust against time delay uncertainties, while stability is guaranteed independently of the amount of the time delay. Although the scattering is a delayindependent approach contrary to the PI controller and the Smith predictor, it performs well compared to the other two controllers even for the initially presumed time delay.

\section{EXPERIMENTAL RESULTS}

The goal of the experiment is the position control of an actuated 1DOF pendulum. The experimental testbed consists of the 1DOF pendulum shown in Fig. 6 connected to a $\mathrm{PC}$ running under RT Linux. The original design of the pendulum can be found in [12].

The DC-motor torque is controlled over a PWM amplifier operated under current control with the reference signal given by a voltage from the $\mathrm{D} / \mathrm{A}$ converter output of the $\mathrm{I} / \mathrm{O}$ board. The position of the lever is measured by an optic pulse incremental encoder and processed by a counter on the I/O board. The control loop including the controller, the scattering transformation and the communication network with constant time delay are implemented as MATLAB/SIMULINK blocksets where standalone realtime code is generated from. All the experiments are performed with a sampling time interval of $T_{A}=1 \mathrm{~ms}$.

The plant is approximated by a transfer function whose parameters are determined experimentally through a square pulse response and consecutive least squares identification. The identified transfer function from the input voltage to the angle of the pendulum is given by $36 /\left(s^{2}+10.15 s\right)$. The output of the plant is chosen to be a linear combination of the position and the velocity in order to reduce oscillations, so the final transfer function is given by

$$
H_{p}(s)=\frac{36}{s+10.15} \frac{0.05 s+1}{s} .
$$

The controller, designed with loop-shaping methods without considering the time delay and the scattering transformation, is given by

$$
H_{c}(s)=\frac{10(s+10.15)}{s+14.311} .
$$

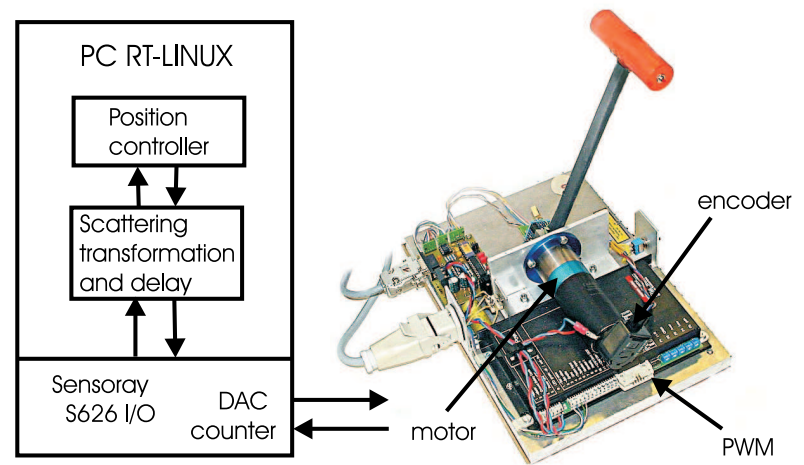

Fig. 6. Experimental testbed 

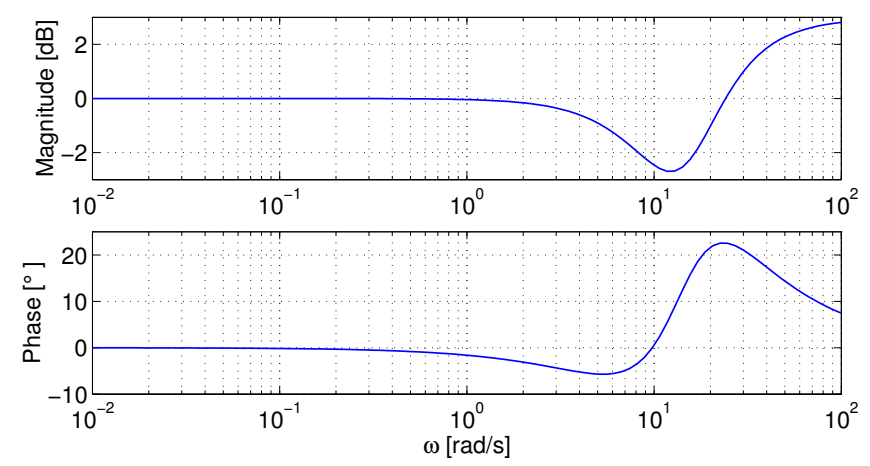

Fig. 7. Frequency response of $\mathrm{K}(\mathrm{s})$

For the above plant and the controller the equation $K(0)=1$ gives $b=0.141$. From the frequency response of $K(s)$ in Fig. 7 it can be seen that $K(s)$ is PR according to the stability condition (7) and up to a frequency of $4 \mathrm{rad} / \mathrm{s} K(\mathrm{~s})$ has a gain of approximately one.

Because of the fact that the controller is biproper, an algebraic loop is created consisting of the controller and the left part of the scattering transformation, which cannot be solved in discrete time. To overcome this obstacle a low pass filter with unit gain and cut-off frequency of $1000 \mathrm{rad} / \mathrm{s}$ is inserted just before the controller. With this filter $K(s)$ remains positive real and further the cut-off frequency of the filter is high enough such that its effect on the response is practically negligible.

In each experiment the pendulum starts from the upright position, the desired position is given by a step function with a final value of $0.2 \mathrm{rad}$. Four different values for the time delay $T \in[75,150,300,600] \mathrm{ms}$ are tested. The results of the experiment are presented in Fig.8 showing that the system is stable and robust to different values of the time delay, as it deteriorates smoothly with the increase of the time delay. Furthermore, the experimental and the simulation results are close. The small difference between the simulation and experimental results as well the steady state error in some of the figures are due to unmodeled dynamics and nonlinearities of the plant such as friction, backlash and gravity forces.

\section{CONCLUSiOnS}

In this paper a delay-independent control approach for networked control systems (NCS) using the scattering transformation is proposed. Compared to conventional control techniques such as the Smith predictor or PI control the stability is guaranteed without knowing the time delay. A novel contribution compared to the known scattering transformation is an extension for non-passive plants. Control design in the presented approach is possible without assuming knowledge about the time delay, the controller can be designed by any design methodology not considering time delay at all and for negligible time delay the performance converges to the non-delay design case. In a simulation example the superior performance of the novel approach is compared to conventional Smith predictor and PI control, in particular for
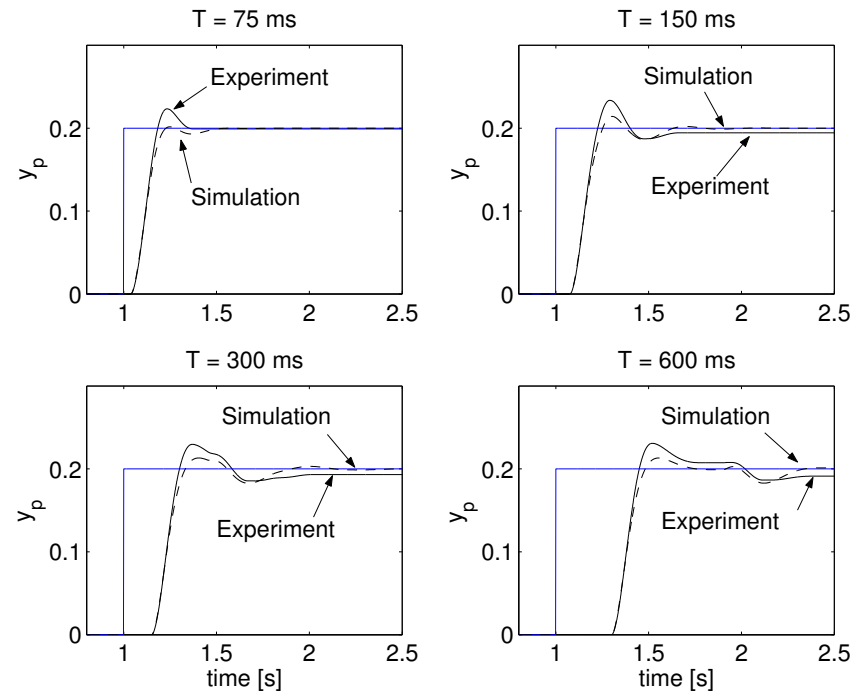

Fig. 8. Experimental results: step responses of the pendulum for various time delays

time delay uncertainties. A pendulum hardware-in-the-loop experiment verifies the efficacy of the proposed approach in experiments. Clearly, the proposed novel approach holds greatest promise for NCS. Future work is to approach timevarying time delay, packet loss, and non-linear systems.

\section{ACKNOWLEDGMENTS}

Detailed comments by the anonymous reviewers are highly appreciated.

\section{REFERENCES}

[1] Zhang, W., Branicky, M.S., and Philips, S.M., "Stability of Network Control Systems," IEEE Control Systems Magazine, , 21(1):84-99, February 2001.

[2] Nilsson, J., "Real-Time Control Systems with Delays," PhD thesis, LUTFD2/TFRT-1049- SE, Department of Automatic Control, Lund Institute of Technology, Lund, Sweden, 1998.

[3] Yu, M., Wang, L., and Chu, T., "An LMI Approach to Robust Stabilization of Networked Control Systems," 16th IFAC World Congress, Prague, Czech Republic, 2005.

[4] Halevi., Y. and Ray, A., "Integrated communication and control systems: Part I Analysis" Journal of Dynamic Systems, Measurement and Control 110, pp 367-373, 1988.

[5] Halevi., Y. and Ray, A., "Integrated communication and control systems: Part II Design Considerations" Journal of Dynamic Systems, Measurement and Control 110, pp 374-381, 1988.

[6] Walsh, G.C., Ye, H. and Bushnell, L., "Stability analysis of Network Control Systems," Proceeding of American Control Conference, San Digo, CA, pp 2876-2880, June 1999.

[7] Tipsuwan, Y. and Chow, M.Y., "Control methodologies in network control systems," Control Engineering Practice 11, pp 1099-1011, 2003.

[8] Chen, J., Gu, K. and Kharitonov, V., "Stability of Time-Delay Systems," Birkhäuser Boston 2003.

[9] Anderson, R.J. and Spong, M.W., "Bilateral Control of Teleoperators with Time Delay," IEEE Transactions on Automatic Control, AC-34, No. 5, pp. 494-501, May, 1989.

[10] Niemeyer, G. and Slotine, J., "Stable Adaptive Teleoperation," Int. J. of Oceanic Engineering, Vol 16, No. 1, pp 152-162, 1991.

[11] H.K. Khalil, "Nonlinear Systems," Prentice Hall, 1996.

[12] Baier, H., Buss, M., Freyberger, F., Hoogen, J., Kammermeier, P. and Schmidt, G., " Distributed PC-Based Haptic, Visual and Acoustic Telepresence System-Experiments in Virtual and Remote Environments," Proceeedings of the IEEE Virtual Reality Conference VR99, Houston, TX, pp. 118-125, 1999. 